\title{
Sliding Mode Control for Visual Servoing of Mobile Robots using a Generic Camera
}

\author{
Héctor M. Becerra and Carlos Sagüés \\ Instituto de Investigación en Ingeniería de Aragón, Universidad de Zaragoza \\ Spain
}

\section{Introduction}

At present, vision sensors represent a very good option for the control of robots since they provide at a low cost a lot of information from the environment. The feasibility of using a vision system as the only source of feedback information has been shown by many approaches (Chaumette \& Hutchinson (2006), Chaumette \& Hutchinson (2007)). Particularly, incorporating machine vision for the control of mobile robots can improve their navigation capabilities (DeSouza \& Kak (2002)). The approach of closing the control loop through a vision system is called visual servoing (VS). The schemes in this control approach can be classified according to the nature of the feedback information. Image data can be used directly in the control loop (image-based schemes IBVS), for instance (Abdelkader et al. (2005), Benhimane \& Malis (2006)), or can be used to compute an estimate of pose parameters (position-based schemes: PBVS) as in (Das et al. (2001), Fontanelli et al. (2009)). Hybrid schemes combining these both approaches have been performed as well (Malis et al. (1999), Fang et al. (2005)).

Most of the current efforts of the research on visual servoing focus on applications of monocular vision. This chapter presents an IBVS approach to drive a wheeled mobile robot equipped with a monocular camera onboard to a desired pose (position and orientation). The desired pose is specified by a target image previously acquired, i.e., the teach-by-showing strategy. In this context, a good way to relate the current and the target view is through a geometric constraint: epipolar geometry or the homography model. A geometric constraint is imposed on images in which there exist correspondences between features (Hartley \& Zisserman (2000)). The information provided by a geometric constraint can be used directly as measurement for output feedback control. Comparing this two-view geometric constraints, the epipolar geometry is a more general approach because it is not constrained to planar objects or planar scenes. Currently, there also exist approaches that use three views (Becerra et al. (2010)).

This chapter focuses on exploiting the epipolar geometry (EG) in an IBVS approach. This constraint has been applied in some works (Basri et al. (1998), Rives (2000), Mariottini et al. (2007), López-Nicolás et al. (2008)). These works deal with the teach-by-showing problem, in which the target pose must be reached using only image data provided from the current and target images. In (Basri et al. (1998)) and (Rives (2000)) are reported visual servoing schemes based on epipolar geometry for manipulators. In the field of mobile robots, an epipolar-based VS approach that takes into account the nonholonomic nature of the robots is introduced in 
(Mariottini et al. (2007)). The resultant motion in this approach steers the robot away from the target while the lateral error is corrected, and after that, the robot moves backward to the target position with a different control. This maneuvers are carried out in order to avoid a singularity problem that is induced by the epipolar geometry. The problem arises when the interaction matrix relating the robot velocities and the rate of change of the epipoles becomes singular. The motion strategy has been improved by driving the robot directly toward the target in the approach presented in (López-Nicolás et al. (2008)), however, in this work, one of the control inputs is not computed when the singularity occurs.

Although some of the previous works claim to achieve good robustness against camera parameters uncertainty, there is not a theoretical support of it. This chapter presents a sliding mode (SM) control law that drives the robot moving always toward the target and deals with the singularity problem. Thanks to the SM control, the robot is able to pass through the singularity caused by the epipolar geometry using bounded control inputs. Moreover, the visual control can be performed even when the initial robot pose is just on the singular point. Additionally, the SM control provides the required robustness to the closed loop control in this type of application. It is particularly important in the case of conventional perspective cameras because the presence of camera calibration uncertainty. This has been tackled through SM control in (Kim et al. (2006)) and (Becerra \& Sagues (2008)). In this chapter, the last work is extended to calibrated omnidirectional images given by a generic camera. This type of camera is considered as an imaging system that approximately obeys the central projection model (Geyer \& Daniilidis (2000)). The use of a generic camera provides the important advantage of keeping the target in the field of view. Wide field of view cameras have been applied for the control of mobile robots, for instance in (Abdelkader et al. (2005)) and (Mariottini \& Prattichizzo (2008)). Although the scheme described herein is a calibrated approach, the benefits of SM control are present in the treatment of the singularity and the robustness against image noise and the uncertainty in a control parameter (the distance between the current and target locations).

The chapter is organized as follows. Section 2 introduces the mobile robot model, summarizes the model of generic cameras and describes the way to estimate the epipolar geometry of this type of cameras. Section 3 details the design procedure for the sliding mode control law. Section 4 presents an stability analysis. Section 5 shows the performance of the closed-loop control system via realistic simulations and finally, Section 6 provides the conclusions.

\section{Mathematical modeling}

\subsection{Robot kinematics}

Many wheeled mobile platforms can be represented as differential-drive robots, whose kinematic model is expressed as the affine system $\dot{\mathbf{z}}=f(t, \mathbf{z})+B(t, \mathbf{z}) \mathbf{u}$. The differential kinematics of the robot to be controlled, in accordance with the frame defined in Fig. 1(a), is as follows

$$
\left[\begin{array}{l}
\dot{x} \\
\dot{y} \\
\dot{\phi}
\end{array}\right]=\left[\begin{array}{cc}
-\sin \phi & 0 \\
\cos \phi & 0 \\
0 & 1
\end{array}\right]\left[\begin{array}{l}
v \\
\omega
\end{array}\right],
$$

where, $\mathbf{z}=(x, y, \phi)^{T}$ represents the state of the robot, $x(t)$ and $y(t)$ are the robot position in the plane and $\phi(t)$ is the orientation. Additionally, $v(t)$ and $\omega(t)$ are the translational and rotational input velocities. The affine model (1) has the particularity that $f(t, \mathbf{z})=\mathbf{0}$. Hence, this is a driftless system (i.e. no motion takes place under zero input, or in control 


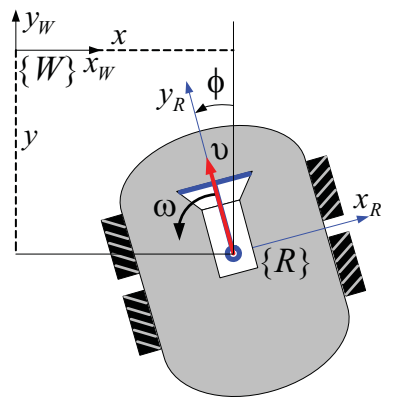

Fig. 1. Upper view of a mobile robot with a camera onboard and important variables of the system, where $\{W\}$ referes to the world frame and $\{R\}$ to the robot frame.

theory concepts, any state is an equilibrium point under zero input). Furthermore, the corresponding linear approximation in any point $\mathbf{z}(t) \in \Re^{3}$ does not have the property of controlability. However, it fulfills the Lie Algebra rank condition (Isidori (1995)), in such a way that controlability can be demonstrated from a nonlinear point of view.

\subsection{Generic camera model}

The constrained field of view of conventional cameras can be enhanced using wide field of view imaging systems such as full view omnidirectional cameras, which capture images as the one in Fig. 2(a). This can be achieved using some optic arrangements that combine mirrors and lens, i.e., catadioptric imaging systems (Fig. 2(b)). These systems use hyperboloidal, paraboloidal or ellipsoidal mirrors and have been well studied in the field of computer vision (Baker \& Nayar (1999)). According to this theory, all of them satisfy the fixed view point constraint. In practice, with a careful construction of the system, it is realistic to assume a central configuration and many robotic applications have proven its effectiveness (Abdelkader et al. (2005), Benhimane \& Malis (2006), Mariottini \& Prattichizzo (2008), Guerrero et al. (2008)).

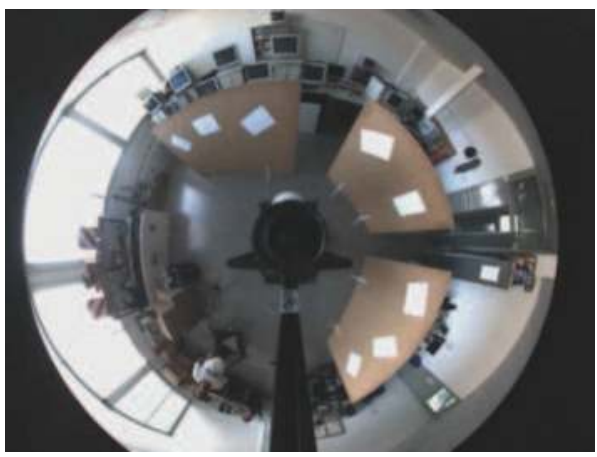

(a) Omnidirectional image

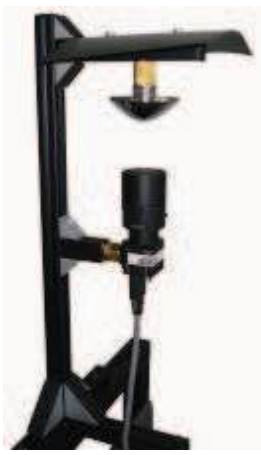

(b) Catadioptric imaging system

Fig. 2. Example of an omnidirectional image and the system to capture this type of images.

It is known that the imaging process performed by conventional and catadioptric cameras can be modeled by a unique representation (Geyer \& Daniilidis (2000)). Such unified 
projection model works properly for imaging systems having a single center of projection (central cameras). Although fisheye cameras do not accomplish such property, some recent experimental results have shown that the unified projection model is able to represent their image formation process with the required accuracy for robotic applications (Courbon et al. (2007)).

The unified projection model describes the image formation as a composition of two central projections (Geyer \& Daniilidis (2000)). The first is a central projection of a 3D point onto a virtual unitary sphere and the second is a perspective projection onto the image plane. According to (Barreto \& Araujo (2005)), this generic model can be parameterized by $(\xi, \lambda)$ which are parameters describing the type of imaging system and by the matrix $\mathbf{K}$ containing the intrinsic parameters

$$
\mathbf{K}_{c}=\left[\begin{array}{ccc}
\alpha_{x} & s & x_{0} \\
0 & \alpha_{y} & y_{0} \\
0 & 0 & 1
\end{array}\right],
$$

where $\alpha_{x}$ and $\alpha_{y}$ represent the focal length of the camera in terms of pixel dimensions in the $x$ and $y$ directions respectively, $s$ is the skew parameter and $\left(x_{0}, y_{0}\right)$ are the coordinates of the principal point.

The parameter $\xi$ encodes the nonlinearities of the image formation in the range $0 \leq \xi \leq 1$ for the cases of catadioptric cameras and $\xi>1$ for fisheye cameras. The parameter $\lambda$ can be seen as a factor for the focal length and it is already included in its estimated value. Thus, the parameter $\xi$ and the camera parameters can be obtained through a calibration process using an algorithm for central catadioptric cameras like the one in (Mei \& Rives (2007)).

The mapping of a point $\mathbb{X}$ in the $3 \mathrm{D}$ world with coordinates $\mathbf{X}=[X, Y, Z]^{T}$ in the camera frame $F_{c}$ resulting in the image point $\mathbf{x}_{i c}$ with homogeneous coordinates $\mathbf{x}_{i c}^{h}$ can be divided into three steps (refer to Fig. 3):

1. The world point is projected onto the unit sphere on a point $\mathbb{X}_{c}$ with coordinates $\mathbf{X}_{c}$ in $F_{c}$, which are computed as $\mathbf{X}_{c}=\mathbf{X} /\|\mathbf{X}\|$.

2. The point coordinates $\mathbf{X}_{c}$ are then changed to a new reference frame $O_{c}$ centered in $O=$ $[0,0,-\xi]^{T}$ and perspectively projected onto the normalized image plane $Z=1-\xi$ :

$$
\begin{aligned}
\mathbf{x}^{h} & =\left[\mathbf{x}^{T}, 1\right]^{T}=[x, y, 1]^{T} \\
& =\left[\frac{X}{Z+\zeta\|\mathbf{X}\|}, \frac{Y}{Z+\zeta\|\mathbf{X}\|}, 1\right]^{T} .
\end{aligned}
$$

3. The image coordinates expressed in pixels are obtained after a collineation $\mathbf{K}$ of the $2 \mathbf{D}$ projected point $\mathbf{x}_{i c}^{h}=\mathbf{K} \mathbf{x}^{h}$.

The matrix $\mathbf{K}$ can be written as $\mathbf{K}=\mathbf{K}_{c} \mathbf{M}$, where $\mathbf{K}_{c}$ has been given in (2) and $\mathbf{M}$ is the following diagonal matrix

$$
\mathbf{M}=\left[\begin{array}{ccc}
\lambda-\xi & 0 & 0 \\
0 & \xi-\lambda & 0 \\
0 & 0 & 1
\end{array}\right]
$$

Notice that, setting $\xi=0$, the general projection model becomes the well known perspective projection model. Images also depend on the extrinsic parameters $\mathbf{C}=(x, y, \phi)$, i.e. the camera pose in the plane relative to a global reference frame. Then an image is denoted by $I(\mathbf{K}, \mathbf{C})$. 


\subsection{Epipolar geometry}

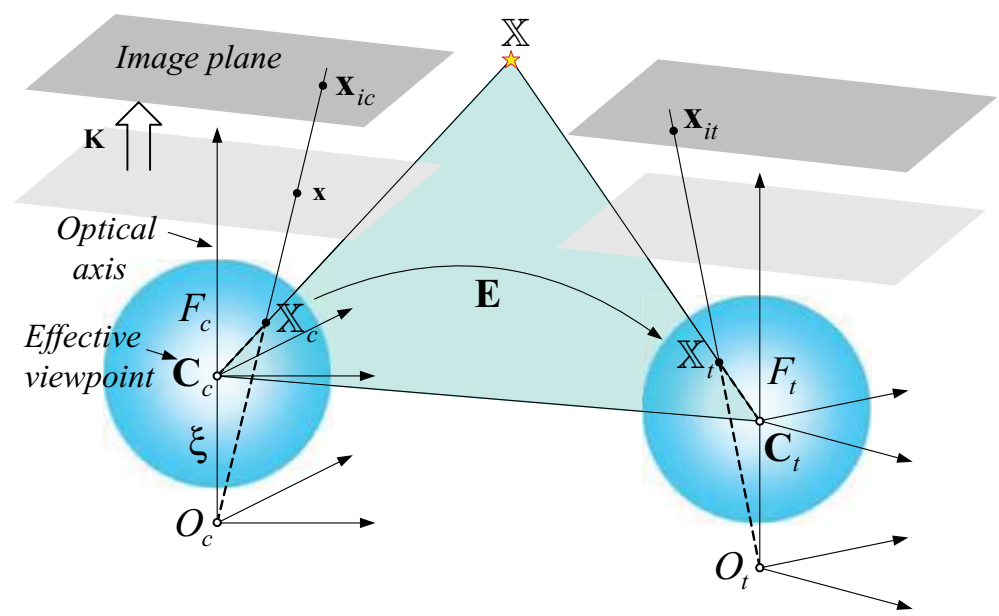

Fig. 3. Generic model of the image formation and epipolar geometry between generic central cameras.

Regarding to Fig. 3, let $\mathbb{X}$ be a 3D point and let $\mathbf{X}_{c}$ and $\mathbf{X}_{t}$ be the coordinates of that point projected onto the unit spheres of the current $F_{c}$ and target frame $F_{t}$. The epipolar plane contains the effective viewpoints of the imaging systems $\mathbf{C}_{c}$ and $\mathbf{C}_{t}$, the $3 \mathrm{D}$ point $\mathbb{X}$ and the points $\mathbb{X}_{c}$ and $\mathbb{X}_{t}$. The coplanarity of these points leads to the well known epipolar constraint

$$
\mathbf{X}_{c}^{T} \mathbf{E} \mathbf{X}_{t}=0,
$$

being $\mathbf{E}$ the essential matrix relating the pair of normalized virtual cameras. Normalized means that the effect of the known calibration matrix has been removed and virtually, the cameras can be represented as perspective. As typical, from this geometry it is possible to compute the epipoles as the points lying on the base line and intersecting the corresponding virtual image plane. Figure 4(a) shows the epipolar geometry for a pair of catadioptric systems and Fig. 4(b) depicts the projection of the epipoles in the produced omnidirectional images. The virtual representation of these imaging systems as perspective cameras is shown in Fig. 4(c) considering the planar motion constraint. A global reference frame centered in the origin $\mathbf{C}_{t}=(0,0,0)$ of the target viewpoint is defined, as well as important parameters. Then, the current camera location with respect to this reference is $\mathbf{C}_{c}=(x, z, \phi)$. Assuming the described framework in Fig. 1, where the camera location coincides with the robot location, the epipoles can be written as a function of the robot state as follows:

$$
\begin{aligned}
e_{c x} & =\alpha_{x} \frac{x \cos \phi+y \sin \phi}{y \cos \phi-x \sin \phi^{\prime}} \\
e_{t x} & =\alpha_{x} \frac{x}{y} .
\end{aligned}
$$

Cartesian coordinates $x$ and $y$ can be expressed as a function of the polar coordinates $d$ and $\psi$ as

$$
x=-d \sin \psi, y=d \cos \psi,
$$




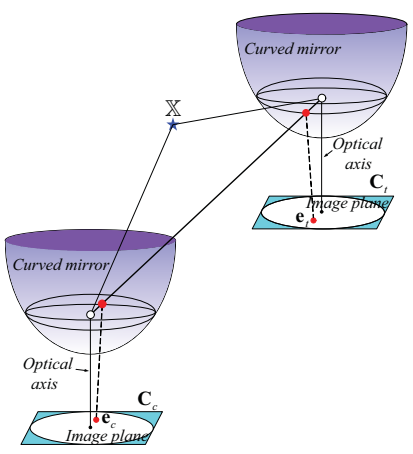

(a) 3D epipolar geometry (EG).

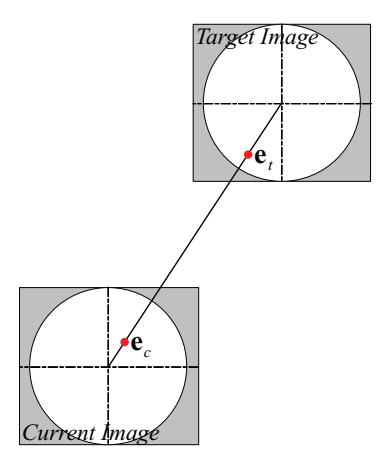

(b) EG in omnidirectional images.

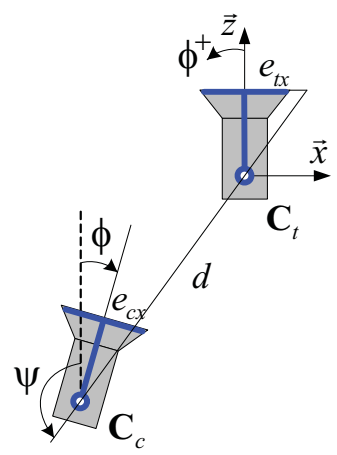

(c) Planar EG framework.

Fig. 4. Generic cameras can be treated as virtual perspective cameras, in which the epipolar geometry is estimated as typical when the points on the unitary sphere are used.

with $\psi=-\arctan \left(e_{t x} / \alpha_{x}\right), \phi-\psi=\arctan \left(e_{c x} / \alpha_{x}\right)$ and $d^{2}=x^{2}+y^{2}$. For the case of normalized cameras $\alpha_{x}=1$ in (5) and in the subsequent equations.

\section{Sliding mode control law}

The goal of this work is to steer a mobile robot to a target pose by using the feedback information provided by the $x$-coordinate of the epipoles for any type of central camera. The visual servoing problem is transformed in a tracking problem for a nonlinear system, where the references for the epipoles are defined. A robust tracking control law under image noise and uncertainty of parameters is designed on the basis of SM control theory. We propose to perform a smooth motion toward the target position by tracking sinusoidal references to drive the epipoles to zero.

The main concern of the proposal is to deal with the singularity problem that arises because the decoupling matrix of the system becomes singular in a point of the state trajectory. This causes the translational velocity to grow unbounded when the system evolves near to that point. This problem is considered by (Mariottini et al. (2007)), where reaching the singular value is avoided during the servoing by using a particular motion strategy. In (López-Nicolás et al. (2008)), one of the control inputs is not computed when the singularity happens. Our strategy is able to pass through the singularity by switching to a bounded SM control law, instead of avoiding to reach to it. Furthermore, this approach can be also used when the initial robot pose is just on the singularity.

Let us define the outputs of the system using the $x$-coordinates of the epipoles for the current image $I_{\mathcal{C}}\left(\mathbf{K}, \mathbf{C}_{2}(t)\right)$ and the target one $I_{t}(\mathbf{K}, \mathbf{0})$. Then, the two-dimensional output of the system is

$$
\mathbf{y}=h(\mathbf{x})=\left[e_{c x}, e_{t x}\right]^{T} .
$$

It can be seen from (5) that if both epipoles are zero implies $x=0, \phi=0$ and $y$ (depth error) may be different to zero. From a control theory point of view this means that, when the epipoles reach to zero the so-called zero dynamics is achieved in the robot system. Zero dynamics is described by a subset of the state space that makes the output to be identically 
zero (Sastry (1999)). Thus, in the particular case of the robot system (1) with output vector (7), this set is given as follows

$$
\begin{aligned}
Z^{*} & =\left\{[x, y, \phi]^{T} \mid e_{c x} \equiv 0, e_{t x} \equiv 0\right\} \\
& =\left\{[0, y, 0]^{T}, y \in \mathbb{R}\right\} .
\end{aligned}
$$

Zero dynamics in this control system makes necessary a second step in which the remaining depth error must be corrected. We address the depth correction by using a constant translational velocity and the stop condition is given by thresholding the image error between corresponding points of the current and the target views. The image error is defined as the mean squared error between the $p$ corresponding image points of the current image and points of the target image, i.e.,

$$
\epsilon=\frac{1}{p} \sum_{j=1}^{p}\left\|\mathbf{x}_{t, j}-\mathbf{x}_{c, j}\right\| .
$$

In order to design the appropriate control law the following tracking error system (r-system) is obtained by using the change of variables $r_{c}=e_{c x}-e_{c x}^{d}, r_{t}=e_{t x}-e_{t x}^{d}$ and the polar coordinates (6).

$$
\left[\begin{array}{c}
\dot{r}_{c} \\
\dot{r}_{t}
\end{array}\right]=\left[\begin{array}{cc}
-\frac{\alpha_{x} \sin (\phi-\psi)}{d \cos ^{2}(\phi-\psi)} & \frac{\alpha_{x}}{\cos ^{2}(\phi-\psi)} \\
-\frac{\alpha_{x} \sin (\phi-\psi)}{d \cos ^{2}(\psi)} & 0
\end{array}\right]\left[\begin{array}{c}
v \\
\omega
\end{array}\right]-\left[\begin{array}{c}
\dot{e}_{c x}^{d} \\
\dot{e}_{t x}^{d}
\end{array}\right] .
$$

The system (10) has the form $\dot{\mathbf{r}}=\mathbf{M}(\phi, \psi) \mathbf{u}-\dot{\mathbf{e}}^{d}$, where $\mathbf{M}(\phi, \psi)$ corresponds to the decoupling matrix and $\dot{\mathbf{e}}^{d}$ represents a known disturbance. It is evident that the decoupling matrix loses rank if $\phi-\psi=n \pi$ with $n \in \mathbb{Z}$. For all the rest of the state space this matrix is invertible, with inverse matrix

$$
\mathbf{M}^{-1}(\phi, \psi)=\frac{1}{\alpha_{x}}\left[\begin{array}{cr}
0 & -\frac{d \cos ^{2}(\psi)}{\sin (\phi-\psi)} \\
\cos ^{2}(\phi-\psi) & -\cos ^{2}(\psi)
\end{array}\right] .
$$

We faced the tracking problem as an stabilization problem of the error system (10).

\subsection{Decoupling-based control law}

Firstly, in order to design a SMC law, we have to define suitable sliding surfaces. The simplest way to do it for the r-system (10) is to use directly the errors as sliding surfaces, in such a way that if there exist switching feedback gains that make the states to evolve in $\mathbf{s}=0$, then the tracking problem is solved. Thus, the sliding surfaces are the following

$$
\mathbf{s}=\left[\begin{array}{l}
s_{c} \\
s_{t}
\end{array}\right]=\left[\begin{array}{l}
r_{c} \\
r_{t}
\end{array}\right]=\left[\begin{array}{c}
e_{c x}-e_{c x}^{d} \\
e_{t x}-e_{t x}^{d}
\end{array}\right] .
$$

Next, the equivalent control method is used to find switching feedback gains to drive the state trajectory to $\mathbf{s}=0$ and to maintain it there. The equivalent control method consists in working out the value of inputs from the equation $\dot{\mathbf{s}}=0$. The so-called equivalent control is then

$$
\mathbf{u}_{e q}=\mathbf{M}^{-1}(\phi, \psi) \dot{\mathbf{e}}^{d}
$$


A decoupling-based SMC law that ensures global stabilization of the r-system has the form $\mathbf{u}_{s m}=\mathbf{u}_{e q}+\mathbf{u}_{\text {disc }}$, where $\mathbf{u}_{\text {disc }}$ is a two-dimensional vector containing switching feedback gains. We propose the simplest form of these gains as follows

$$
\mathbf{u}_{\text {disc }}=\mathbf{M}^{-1}(\phi, \psi)\left[\begin{array}{l}
-k_{c}^{s m} \operatorname{sign}\left(s_{c}\right) \\
-k_{t}^{s m} \operatorname{sign}\left(s_{t}\right)
\end{array}\right],
$$

where $k_{c}^{s m}>0$ and $k_{t}^{s m}>0$ are control gains. Although $\mathbf{u}_{s m}$ can achieve global stabilization of the r-system, it needs high gains and, consequently, the state trajectory may not reach the sliding surfaces in a smooth way. This could cause a non-smooth behavior in the robot state that is not valid in real situations. We add a pole placement term in the control law to alleviate this problem

$$
\mathbf{u}_{p p}=\mathbf{M}^{-1}(\phi, \psi)\left[\begin{array}{cc}
-k_{c} & 0 \\
0 & -k_{t}
\end{array}\right]\left[\begin{array}{l}
s_{c} \\
s_{t}
\end{array}\right],
$$

where $k_{c}>0$ and $k_{t}>0$ are control gains. Finally, the complete SMC law that achieves robust global stabilization of the system (10) is as follows

$$
\mathbf{u}_{d b}=\left[\begin{array}{c}
v_{d b} \\
\omega_{d b}
\end{array}\right]=\mathbf{u}_{e q}+\mathbf{u}_{d i s c}+\mathbf{u}_{p p}
$$

\subsection{Bounded control law}

The control law (16) utilizes the decoupling matrix and it presents the singularity problem for the condition $e_{c x}=0(\phi-\psi=n \pi$ with $n \in \mathbb{Z})$, which means that the camera axis of the robot at its current pose is aligned with the baseline. We can note from (11) that the singularity only affects the translational velocity computation. In order to pass through this singularity we propose to commute to a direct sliding mode controller when $\phi-\psi$ is near to $n \pi$. This kind of controller has been studied for output tracking through singularities (Hirschorn (2002)). The direct sliding mode controller is as follows

$$
\mathbf{u}_{b}=\left[\begin{array}{c}
v_{b} \\
\omega_{b}
\end{array}\right]=\left[\begin{array}{c}
-M \operatorname{sign}\left(s_{t} b(\phi, \psi)\right) \\
-\operatorname{Nign}\left(s_{c}\right)
\end{array}\right],
$$

where $M$ and $N$ are suitable gains and $b(\phi, \psi)$ is a function that describes the change in sign of the translational velocity when the state trajectory crosses the singularity. We can find out this function from (10) as follows

$$
\begin{aligned}
& \dot{r}_{c}=b_{1}(\phi, \psi) v+b_{2}(\phi, \psi) \omega-\dot{e}_{c x}^{d}, \\
& \dot{r}_{t}=b_{3}(\phi, \psi) v-\dot{e}_{t x}^{d}
\end{aligned}
$$

where $b_{1}=-\frac{\alpha_{x} \sin (\phi-\psi)}{d \cos ^{2}(\phi-\psi)}, b_{2}=\frac{\alpha_{x}}{\cos ^{2}(\phi-\psi)}, b_{3}=-\frac{\alpha_{x} \sin (\phi-\psi)}{d \cos ^{2}(\psi)}$. According to that, $b_{2}$ is always positive, and $\operatorname{sign}\left(b_{1}\right)=\operatorname{sign}\left(b_{3}\right)=\operatorname{sign}(-\sin (\phi-\psi))$. Hence,

$$
b(\phi, \psi)=-\sin (\phi-\psi) .
$$

The control law (17) with $b(\phi, \psi)$ as in (19) locally stabilizes the system (10) and is always bounded. 


\subsection{Desired references of the epipoles}

As main requirement, the references to track must provide a smooth zeroing of the epipoles starting from their initial values. Figure 5(a) shows two configurations of robot locations for cases in which sign $\left(e_{23}\right) \neq \operatorname{sign}\left(e_{32}\right)$. From these conditions, the epipoles are naturally reduced to zero as the robot moves smoothly toward the target. Because of the nonholomic motion constraint, any direct path reaching the target implies $\operatorname{sign}\left(e_{23}\right) \neq \operatorname{sign}\left(e_{32}\right)$. Therefore, locations starting sign $\left(e_{23}\right)=\operatorname{sign}\left(e_{32}\right)$ need to be controlled to the situation of $\operatorname{sign}\left(e_{23}\right) \neq \operatorname{sign}\left(e_{32}\right)$. This allows getting an adequate orientation from the very beginning (Fig. 5(b)) in order to be able to align the robot with the target at the end of the first step. It is worth emphasizing that this initial rotation is autonomously carried out through the control inputs given by the described controllers. Thus, we define the following desired trajectories, which are always opposite in sign each other

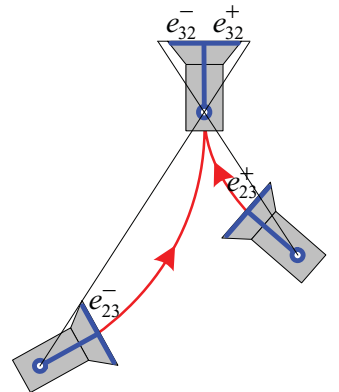

(a) Condition where $\operatorname{sign}\left(e_{t x}\right) \neq \operatorname{sign}\left(e_{c x}\right)$

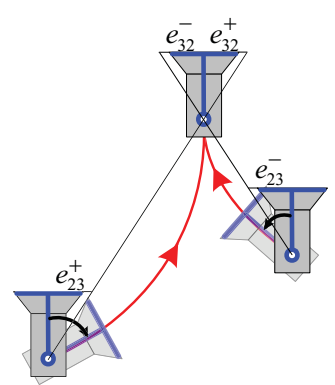

(b) Condition where sign $\left(e_{t x}\right)=\operatorname{sign}\left(e_{c x}\right)$

Fig. 5. Motion strategy for different initial locations. For the cases in (a) a direct motion toward the target is carried out and for those in (b), the robot rotates initially to reach the same condition as in (a).

$$
\begin{aligned}
& e_{c x}^{d}(t)=S \frac{e_{c x}(0)}{2}\left(1+\cos \left(\frac{\pi}{\tau} t\right)\right), \quad 0 \leq t \leq \tau \\
& e_{c x}^{d}(t)=0, \quad \tau<t<\infty \\
& e_{t x}^{d}(t)=\frac{e_{t x}(0)}{2}\left(1+\cos \left(\frac{\pi}{\tau} t\right)\right), \quad 0 \leq t \leq \tau \\
& e_{t x}^{d}(t)=0, \quad \tau<t<\infty
\end{aligned}
$$

where $S=-\operatorname{sign}\left(e_{c x}(0) e_{t x}(0)\right)$ and $T$ is the time to reach epipoles to zero. We highlight that since our scheme is an IBVS approach, the desired trajectories in the image space play an important role in the resulting Cartesian path. In fact, by only changing the trajectory for the target epipole (related to the translational velocity) is possible to run our approach for car-like robots. Thus, the references may also be used to constraint the target in the field of view. However, as mentioned previously, this problem does not appear when using omnidirectional vision. 


\section{Stability analysis}

In this section, the stability of both proposed sliding mode control laws is analyzed. Given that the relationship between zeroing the epipoles and the robot state has been established through the zero dynamics, we focus on demonstrating the convergence property of the tracking control law as stated in the following proposition.

Proposition 1. A commuted control law that combines the decoupling-based control (16) by switching to the bounded control (17) whenever $|\phi-\psi|<n \pi+T_{h}$, where $T_{h}$ is a suitable threshold value and $n \in \mathbb{Z}$, achieves global stabilization of the system (10). Moreover, global stabilization is achieved even with uncertainty in parameters.

Proof. Stabilization of the system (10) is proved by showing that the sliding surfaces can be reached in a finite time (existence conditions of sliding modes). Let be the natural Lyapunov function for a sliding mode controller

$$
V=V_{1}+V_{2}, \quad V_{1}=\frac{1}{2} s_{c}^{2}, \quad V_{2}=\frac{1}{2} s_{t}^{2},
$$

which accomplish $V\left(s_{c}=0, s_{t}=0\right)=0$ and $V>0$ for all $s_{c} \neq 0, s_{t} \neq 0$.

$$
\dot{V}=\dot{V}_{1}+\dot{V}_{2}=s_{\mathcal{c}} \dot{s}_{c}+s_{t} \dot{s}_{t} .
$$

We analyze each term of (22) for the decoupling-based controller (16)

$$
\begin{aligned}
& \dot{V}_{1}=s_{c}\left(-\frac{\alpha_{x}}{\alpha_{x_{e}}}\left(k_{c}^{s m} \operatorname{sign}\left(s_{c}\right)+k_{c} s_{c}\right)+A\right)=-\left(\frac{\alpha_{x}}{\alpha_{x_{e}}}\left(k_{c}^{s m}\left|s_{c}\right|+k_{c} s_{c}^{2}\right)-s_{c} A\right), \\
& \dot{V}_{2}=s_{t}\left(-\frac{\alpha_{x} d_{e}}{\alpha_{x_{e}} d}\left(k_{t}^{s m} \operatorname{sign}\left(s_{t}\right)+k_{t} \xi_{t}\right)+B\right)=-\left(\frac{\alpha_{x} d_{e}}{\alpha_{x_{e}} d}\left(k_{t}^{s m}\left|s_{t}\right|+k_{t} s_{t}^{2}\right)-s_{t} B\right),
\end{aligned}
$$

where $A=\frac{\alpha_{x}}{\alpha_{x_{e}}}\left(\frac{d_{e}}{d}-1\right)\left(\dot{e}_{t x}^{d}-k_{t}^{\operatorname{sm}} \operatorname{sign}\left(s_{t}\right)-k_{t} s_{t}\right) \frac{\cos ^{2}(\psi)}{\cos ^{2}(\phi-\psi)}+\left(\frac{\alpha_{x}}{\alpha_{x_{e}}}-1\right) \dot{e}_{c x}^{d}$, $B=\left(\frac{\alpha_{x} d_{e}}{\alpha_{x_{e}}}-1\right) \dot{e}_{t x}^{d}$, and $\alpha_{x_{e}}, d_{e}$ represent estimated values for the corresponding system parameter. We can see that

$$
\begin{aligned}
& \dot{V}_{1} \leq-\left(\frac{\alpha_{x}}{\alpha_{x_{e}}}\left(k_{c}^{s m}+k_{c}\left|s_{c}\right|\right)-|A|\right)\left|s_{c}\right|, \\
& \dot{V}_{2} \leq-\left(\frac{\alpha_{x} d_{e}}{\alpha_{x_{e}} d}\left(k_{t}^{s m}+k_{t}\left|s_{t}\right|\right)-|B|\right)\left|s_{t}\right| .
\end{aligned}
$$

$\dot{V}_{1}$ and $\dot{V}_{2}$ are negative definite iff the following inequalities are guaranteed for all $s_{c} \neq 0$, $s_{t} \neq 0$

$$
\begin{aligned}
& k_{c}^{s m}+k_{c}\left|s_{c}\right|>\frac{\alpha_{x_{e}}}{\alpha_{x}}|A|, \\
& k_{t}^{s m}+k_{t}\left|s_{t}\right|>\frac{\alpha_{x_{e}} d}{\alpha_{x} d_{e}}|B| .
\end{aligned}
$$

Therefore, $\dot{V}<0$ iff both inequalities (23) are fulfilled. Global convergence to the sliding surfaces can be achieved regardless of uncertainty in parameters. 
Now, let us develop the existence conditions of sliding modes for the bounded controller (17). The same Lyapunov function (21) is used. For each term of (22) after some basic operations we have

$$
\begin{aligned}
& \dot{V}_{1}=-N \frac{\alpha_{x}}{\cos ^{2}(\phi-\psi)}\left|s_{c}\right|-s_{c} \dot{e}_{c x}^{d}-s_{c} C, \\
& \dot{V}_{2}=-M \frac{\alpha_{x}|b(\phi, \psi)|}{d \cos ^{2}(\psi)}\left|s_{t}\right|-s_{t} \dot{e}_{t x}^{d},
\end{aligned}
$$

where $C=M \frac{\alpha_{x}|b(\phi, \psi)|}{d \cos ^{2}(\phi-\psi)} \operatorname{sign}\left(s_{t}\right)$ and $b(\phi, \psi)$ is given in (19). We can see that

$$
\begin{aligned}
& \dot{V}_{1} \leq-\left(N \frac{\alpha_{x}}{\cos ^{2}(\phi-\psi)}-\left|\dot{e}_{c x}^{d}\right|-|C|\right)\left|s_{c}\right| \\
& \dot{V}_{2} \leq-\left(M \frac{\alpha_{x}|b(\phi, \psi)|}{d \cos ^{2}(\psi)}-\left|\dot{e}_{t x}^{d}\right|\right)\left|s_{t}\right|
\end{aligned}
$$

$\dot{V}_{1}$ and $\dot{V}_{2}$ are negative definite iff the following inequalities are assured for all $s_{c} \neq 0, s_{t} \neq 0$

$$
\begin{aligned}
& N>\frac{\cos ^{2}(\phi-\psi)}{\alpha_{x}}\left(|C|+\left|\dot{e}_{c x}^{d}\right|\right), \\
& M>\frac{d \cos ^{2}(\psi)}{\alpha_{x}|b(\phi, \psi)|}\left|\dot{e}_{t x}^{d}\right| .
\end{aligned}
$$

Therefore, $\dot{V}<0$ iff both inequalities (24) are fulfilled. The bounded controller does not need any information of system parameters and thus, its robustness is implicit.

According to the existence conditions of sliding modes, the bounded controller (17) is able to locally stabilize the system (10); its region of attraction is bigger as long as the control gains $M$ and $N$ are higher. Nevertheless, this controller can not achieve the smooth behavior demanded for real situations and it is only used to cross the singularity. Due to the control strategy commutes between two switching control laws and each one acts inside of its region of attraction, respectively, the commutation between the control laws does not affect the stability of the control system. The decoupling-based controller ensures entering to the region of attraction of the bounded one.

Once sliding surfaces are reached for any case of SMC law, the system's behavior is independent of uncertainties and disturbances. It is clear that uncertainties in the system (10) fulfill the matching condition and then, robustness of the control system is accomplished.

\section{Performance evaluation}

The evaluation of the approach has been carried out through realistic simulations. These simulations have been performed in Matlab with a sampling time of $100 \mathrm{~ms}$. The results show that the main objective of driving the robot to a desired pose $\left(\left(0,0,0^{\circ}\right)\right.$ in all the cases $)$ is attained in spite of passing through the singularity that occurs in the first step for some initial poses, and moreover, the task is accomplished even when the robot starts exactly in a singular pose. The good performance of the approach with noise in the images is also reported. 
Regarding to the parameters of the control law, the initial distance between the current and target locations $\left(d_{e}\right)$ is fixed to $10 \mathrm{~m}$ in all the cases. The threshold to switch to the bounded control law $\left(T_{h}\right)$ is set to $0.03 \mathrm{rad}$. Related to the control gains, they are set to $k_{c}=2, k_{t}=1$, $k_{c}^{s m}=0.2, k_{t}^{s m}=0.2, M=0.1$ and $N=0.06$. Synthetic images of size $640 \times 480$ pixels are used to estimate the epipoles at each instant time. These images are obtained by using adequate camera parameters in the generic model of Section 2.2. We present results with hypercatadioptric, paracatadioptric and also fisheye cameras, which can be approximately represented with the same model (Courbon et al. (2007)).

The simulations are carried out for four different initial locations: $\left(-5,-9,-50^{\circ}\right),\left(-4,-14,0^{\circ}\right)$, $\left(8,-16,10^{\circ}\right)$ and $\left(2.5,-12,11.77^{\circ}\right)$ and consequently, the fixed value of $d_{e}$ represents a significative uncertainty in the control parameter. In spite of that, the good behavior of the approach can be seen in the image space through the pictures in Fig. 6 . This figure shows the motion of the point features for the different types of synthetic images used. We can notice that the points of the images at the end of the motion (marker " $\times$ ") are practically the same as the ones in the target images (marker “ $\mathrm{O}^{\prime \prime}$ ).

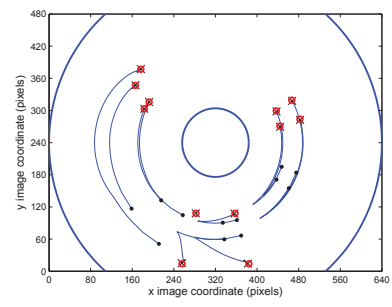

(a) $\left(-4,-14,0^{\circ}\right)$ Paracatad.

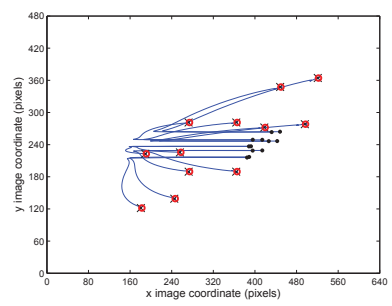

(b) $\left(8,-16,10^{\circ}\right)$ Fisheye.

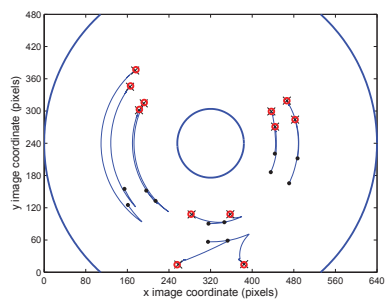

(c) $\left(2.5,-12,11.77^{\circ}\right)$ Hypercatad.

Fig. 6. Behavior of the approach in the image space for different omnidirectional images.

Figure 7(a) shows the resultant paths and the evolution of the epipoles for each one of the initial locations. The case $\left(-5,-9,-50^{\circ}\right)$ corresponds to an initial location from where the robot can exert a direct navigation to the target and has been tested using a hypercatadioptric camera. In the cases $\left(-4,-14,0^{\circ}\right)$ and $\left(8,-16,10^{\circ}\right)$, the robot starts with $\operatorname{sign}\left(e_{c x}\right)=\operatorname{sign}\left(e_{t x}\right)$ and by driving the epipoles to the desired trajectories, $e_{c x}$ changes its sign during the first seconds (Fig. 7(b)). It causes a rotation of the robot, and then, it begins a direct motion toward the target. These cases are tested using paracatadiotric and fisheye cameras respectively.

The initial location $\left(2.5,-12,11.77^{\circ}\right)$, tested with hypercatadioptric images, corresponds to a special case where the state trajectory just starts on the singularity $e_{c x}=0$. The line from the initial position to the target shows that the camera axis is aligned with the baseline for this location. When the robot starts just on the singularity, we assign a suitable amplitude to the desired trajectory for the current epipole. Given that $|\phi-\psi|$ is less than the threshold, the bounded controller takes the system out of the singularity and then, the epipoles evolve as shown in Fig. 7(b). In all the cases both epipoles reach to zero in $\tau=60 \mathrm{~s}$, which is fixed through the desired trajectories.

From the graphics of the epipoles, it can be seen that the state trajectory crosses the singularity $e_{\mathcal{C} x}=0$ for the initial locations $\left(-4,-14,0^{\circ}\right)$ and $\left(8,-16,10^{\circ}\right)$. The behavior of the robot state is presented in Fig. 8(a) for the former case. This is obtained using the bounded input velocities of Fig. 8(b). It is worth noting that the control inputs are maintained bounded even when the epipoles are close to zero after $45 \mathrm{~s}$, which ensures entire correction of orientation and lateral position. It takes approximately $3 \mathrm{~s}$ more to correct the remaining depth error using a constant 


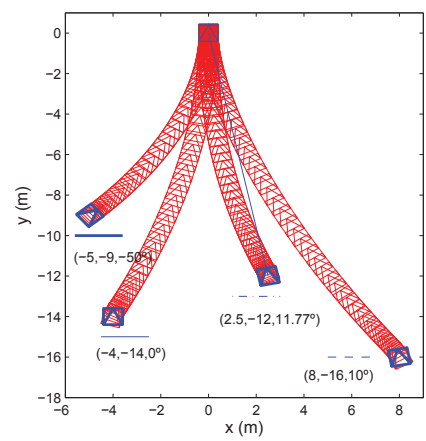

(a) Paths on the $x-y$ plane.
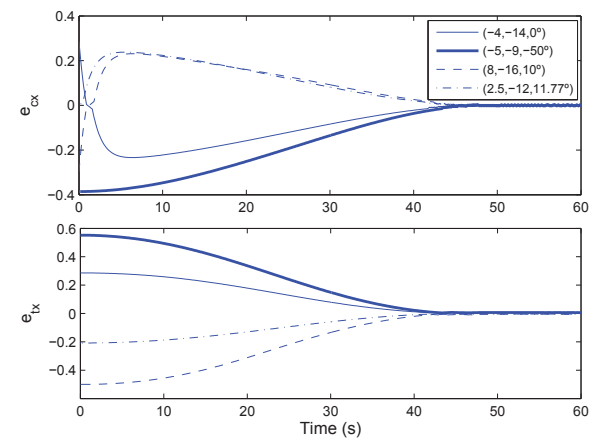

(b) Current and target epipoles.

Fig. 7. Behavior of the approach in the Cartesian space and evolution of epipoles for different initial locations.

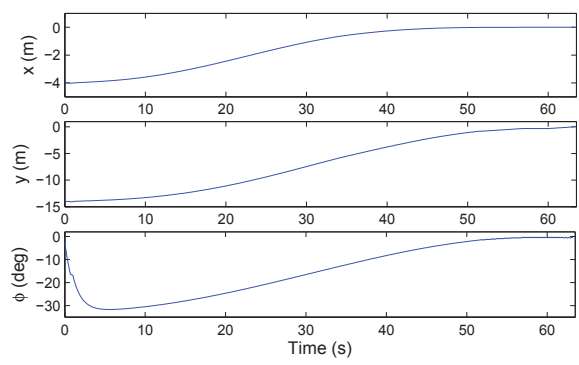

(a) Evolution of the robot state.
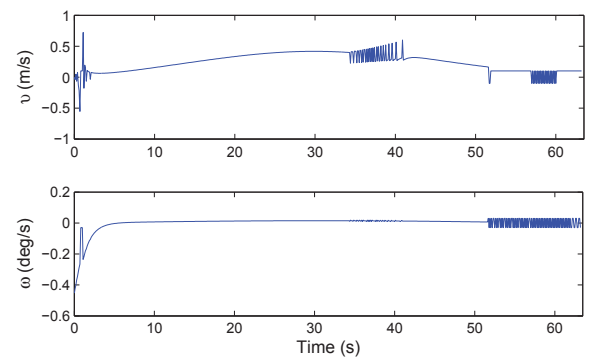

(b) Input velocities.

Fig. 8. Evolution of the position and orientation of the robot and the velocities given by the sliding mode control law for a case where the singularity is crossed, $\left(-4,-14,0^{\circ}\right)$ of Fig. 7 .

translational velocity $v=0.1 \mathrm{~m} / \mathrm{s}$ in this case, but this time may be different for each initial location with the same velocity. The stop condition is given by thresholding the mean squared error (9) between the corresponding image points of the current image and the points of the target image.

Finally, Fig. 9(a) shows the performance of the approach under image noise for the initial location $\left(5,-13,15^{\circ}\right)$. An image noise with standard deviation of 0.5 pixels has been added and the time to reach the alignment with the target is set to $\tau=80 \mathrm{~s}$. During the remaining $9 \mathrm{~s}$, depth correction is carried out by using a constant translational velocity and then, each one of the state variables reaches zero (Fig. 9(b)). It is clear the presence of the noise in the motion of the image points in Fig. 9(c). It can be seen in Fig. 10(a) that the estimated epipoles are more affected by the noise as the robot approaches to the target and eventually it turns out to be unstable (problem of short baseline). However, after $80 \mathrm{~s}$ only the sign of $e_{c x}$ is used to compute the rotational velocity that keeps the robot aligned to the target (Fig. 10(b)).

According to these results when testing the performance of the proposed visual control scheme, the use of SM control provides good benefits in order to solve the singularity problem and robustness against image noise. Additionally, it is worth noting that the target location is always reached with an accuracy in the order of centimeters for position and negligible 


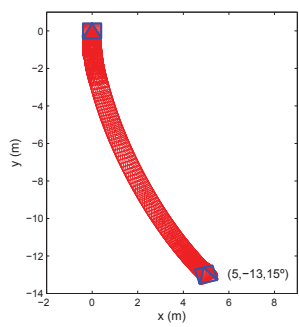

(a) Path on the plane.

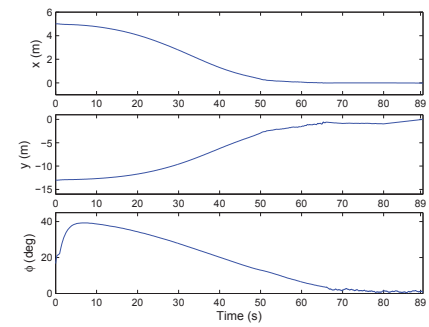

(b) Evolution of the robot state.

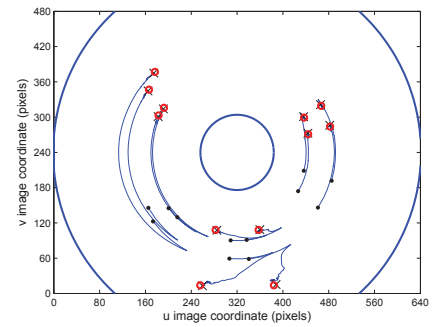

(c) Motion of the image points.

Fig. 9. Robustness under image noise using a hypercatadioptric imaging system.

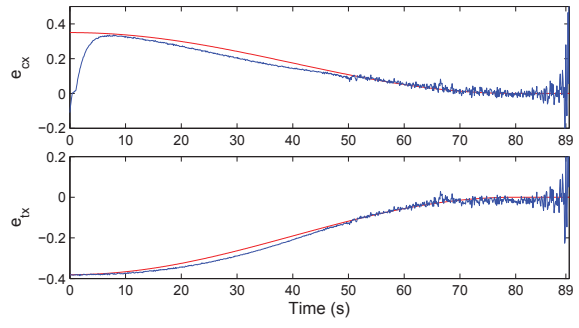

(a) Evolution of the epipoles.

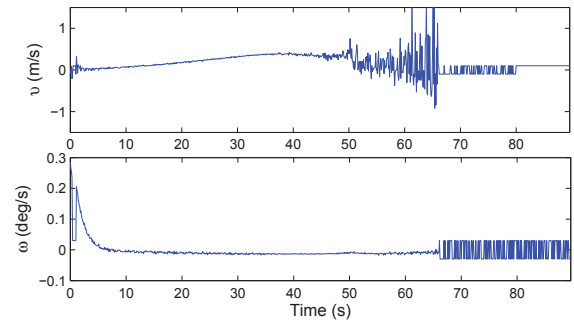

(b) Input velocities.

Fig. 10. Performance of the reference tracking and the velocities given by the sliding mode control law for the servoing task of Fig. 9.

orientation error. This is achieved in spite of the uncertainty in the distance between the current and the target locations $(d)$. As mentioned before, it is enough to fix this value in the controller thanks to the robustness of the control law. We claim that the second phase regarding to depth correction may be carried out exploiting also the information provided by the epipolar geometry. This could be a way to avoid the switching to a totally different approach for depth correction.

\section{Conclusions}

In this chapter, a robust control law to perform image-based visual servoing for differential-drive mobile robots has been presented. The visual control utilizes the usual teach-by-showing strategy, in which the desired location is specified by a target image previously acquired. The mobile robot is driven toward the target by comparing a set of visual features in the current view of the onboard camera and those on the target image. The visual features are gathered through the epipolar geometry and exploited in a sliding mode control law, which provides good robustness against image noise and uncertainty in camera parameters.

The major contribution of this work is the validity of the approach for generic imaging systems. This extends the applicability of the proposed control scheme given that a generic camera allows a major maneuverability of the robot than a conventional camera because its wide field of view. Additionally, the use of sliding mode control allows to solve the problem of passing through a singularity induced by the epipoles, maintaining bounded inputs. Furthermore, the visual control accomplishes its goal even when the robot starts on 
the singularity. The good performance of the approach has been evaluated through realistic simulations using virtual images.

\section{Acknowledgment}

This work has been supported by project MICINN DPI 2009-08126 and grants of Banco Santander-Universidad de Zaragoza and Conacyt-Mexico.

\section{References}

Abdelkader, H. H., Mezouar, Y., Andreff, N. \& Martinet, P. (2005). Image-based control of mobile robot with central catadioptric cameras, IEEE International Conference on Robotics and Automation, pp. 3522-3527.

Baker, S. \& Nayar, S. K. (1999). A theory of single-viewpoint catadioptric image formation, International Journal of Computer Vision 35(2): 175-196.

Barreto, J. P. \& Araujo, H. (2005). Geometric properties of central catadioptric line images and their application in calibration, IEEE Transactions on Pattern Analysis and Machine Intelligence 27(8): 1327-1333.

Basri, R., Rivlin, E. \& Shimshoni, I. (1998). Visual homing: Surfing on the epipoles, IEEE Conference on Computer Vision, pp. 863-869.

Becerra, H. M., López-Nicolás, G. \& Sagues, C. (2010). Omnidirectional visual control of mobile robots based on the 1D trifocal tensor, Robotics and Autonomous Systems 58(6): 796-808.

Becerra, H. M. \& Sagues, C. (2008). A sliding mode control law for epipolar visual servoing of differential-drive robots, IEEE/RSJ International Conference on Intelligent Robots and Systems, pp. 3058-3063.

Benhimane, S. \& Malis, E. (2006). A new approach to vision-based robot control with omni-directional cameras, IEEE International Conference on Robotics and Automation, pp. 526-531.

Chaumette, F. \& Hutchinson, S. (2006). Visual servo control Part I: Basic approaches, IEEE Robotics and Automation Magazine 13(4): 82-90.

Chaumette, F. \& Hutchinson, S. (2007). Visual servo control Part II: Advance approaches, IEEE Robotics and Automation Magazine 14(1): 109-118.

Courbon, J., Mezouar, Y., Eck, L. \& Martinet, P. (2007). A generic fisheye camera model for robotics applications, IEEE/RSJ International Conference on Intelligent Robots and Systems, pp. 1683-1688.

Das, A. K., Fierro, R., Kumar, V., Southall, B., Spletzer, J. \& Taylor, C. J. (2001). Real-time vision-based control of a nonholonomic mobile robot, IEEE/RSJ International Conference on Intelligent Robots and Systems, pp. 1714-1718.

DeSouza, G. \& Kak, A. (2002). Vision for mobile robot navigation: A survey, IEEE Transactions on Pattern Analysis and Machine Intelligence 24(2): 237-267.

Fang, Y., Dixon, W. E., Dawson, D. M. \& Chawda, P. (2005). Homography-based visual servo regulation of mobile robots, IEEE Transactions on Systems, Man, and Cybernetics, Part B 35(5): 1041-1050.

Fontanelli, D., Danesi, A., Belo, F. A. W., Salaris, P. \& Bicchi, A. (2009). Visual servoing in the large, The International Journal of Robotics Research 28(6): 802-814.

Geyer, C. \& Daniilidis, K. (2000). An unifying theory for central panoramic systems and practical implications, European Conference on Computer Vision, pp. 445-461. 
Guerrero, J., Murillo, A. \& Sagüés, C. (2008). Localization and matching using the planar trifocal tensor with bearing-only data, IEEE Transactions on Robotics 24(2): 494-501.

Hartley, R. \& Zisserman, A. (2000). Multiple View Geometry in Computer Vision, Cambridge University Press, Cambridge.

Hirschorn, R. M. (2002). Output tracking through singularities, IEEE Conference on Decision and Control, pp. 3843-3848.

Isidori, A. (1995). Nonlinear Control Systems, Springer, Great Britain.

Kim, J. K., Kim, D. W., Choi, S. J. \& Won, S. C. (2006). Image-based visual servoing using sliding mode control, SICE-ICASE International Joint Conference, pp. 4996-5001.

López-Nicolás, G., Sagüés, C., Guerrero, J., Kragic, D. \& Jensfelt, P. (2008). Switching visual control based on epipoles for mobile robots, Robotics and Autonomous Systems 56(7): 592-603.

Malis, E., Chaumette, F. \& Boudet, S. (1999). 2 1/2 D visual servoing, IEEE Transactions on Robotics and Automation 15(2): 234-246.

Mariottini, G. L., Oriolo, G. \& Prattichizzo, D. (2007). Image-based visual servoing for nonholonomic mobile robots using epipolar geometry, IEEE Transactions on Robotics 23(1): 87-100.

Mariottini, G. L. \& Prattichizzo, D. (2008). Image-based visual servoing with central catadioptric cameras, The International Journal of Robotics Research 27(1): 41-56.

Mei, C. \& Rives, P. (2007). Single view point omnidirectional camera calibration from planar grids, IEEE International Conference on Robotics and Automation, pp. 3945-3950.

Rives, P. (2000). Visual servoing based on epipolar geometry, IEEE/RSJ International Conference on Intelligent Robots and Systems, pp. 602-607.

Sastry, S. (1999). Nonlinear Systems: Analysis, Stability and Control, Springer, New York. 


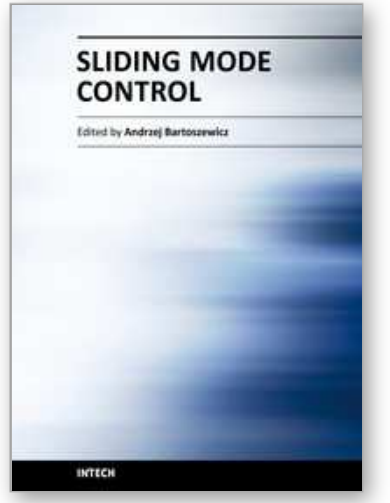

\author{
Sliding Mode Control \\ Edited by Prof. Andrzej Bartoszewicz
}

ISBN 978-953-307-162-6

Hard cover, 544 pages

Publisher InTech

Published online 11, April, 2011

Published in print edition April, 2011

The main objective of this monograph is to present a broad range of well worked out, recent application studies as well as theoretical contributions in the field of sliding mode control system analysis and design. The contributions presented here include new theoretical developments as well as successful applications of variable structure controllers primarily in the field of power electronics, electric drives and motion steering systems. They enrich the current state of the art, and motivate and encourage new ideas and solutions in the sliding mode control area.

\title{
How to reference
}

In order to correctly reference this scholarly work, feel free to copy and paste the following:

Héctor M. Becerra and Carlos Sagüés (2011). Sliding Mode Control for Visual Servoing of Mobile Robots using a Generic Camera, Sliding Mode Control, Prof. Andrzej Bartoszewicz (Ed.), ISBN: 978-953-307-162-6, InTech, Available from: http://www.intechopen.com/books/sliding-mode-control/sliding-mode-control-for-visualservoing-of-mobile-robots-using-a-generic-camera

\section{INTECH}

open science | open minds

\section{InTech Europe}

University Campus STeP Ri

Slavka Krautzeka 83/A

51000 Rijeka, Croatia

Phone: +385 (51) 770447

Fax: +385 (51) 686166

www.intechopen.com

\section{InTech China}

Unit 405, Office Block, Hotel Equatorial Shanghai

No.65, Yan An Road (West), Shanghai, 200040, China

中国上海市延安西路 65 号上海国际贵都大饭店办公楼 405 单元

Phone: +86-21-62489820

Fax: +86-21-62489821 
(C) 2011 The Author(s). Licensee IntechOpen. This chapter is distributed under the terms of the Creative Commons Attribution-NonCommercialShareAlike-3.0 License, which permits use, distribution and reproduction for non-commercial purposes, provided the original is properly cited and derivative works building on this content are distributed under the same license. 